\section{Experiencias de mujeres mexicanas migrantes indocumentadas en California, Estados Unidos, en su acceso a los servicios de salud sexual y reproductiva: estudio de caso}

\author{
Experiences of undocumented Mexican migrant \\ women when accessing sexual and reproductive \\ health services in California, USA: a case study \\ Experiências de mulheres mexicanas migrantes \\ sem documentação na Califórnia, Estados Unidos, \\ no acesso aos serviços de saúde sexual e \\ reprodutiva: estudo de caso
}

Natalia Deeb-Sossa 1 Claudia Díaz Olavarrieta 2 Clara Juárez-Ramírez 2 Sandra G. García 3 Aremis Villalobos 2

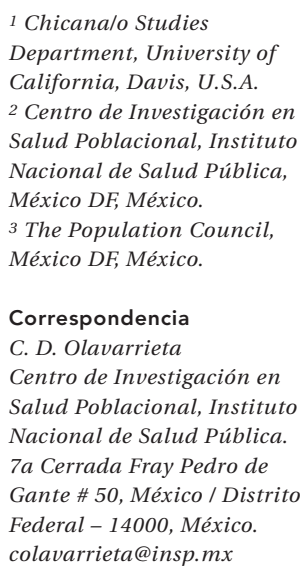

Correspondencia C. D. Olavarrieta Centro de Investigación en Salud Poblacional, Instituto Nacional de Salud Pública. 7 a Cerrada Fray Pedro de Gante \# 50, México / Distrito Federal-14000, México. colavarrieta@insp.mx

\begin{abstract}
This study focuses on the experience of Mexican women migrants in California, USA, with the use of formal health services for sexual and reproductive health issues. The authors used a qualitative interpretative approach with life histories, interviewing eight female users of healthcare services in California and seven key informants in Mexico and California. There were three main types of barriers to healthcare: immigration status, language, and gender. Participants reported long waiting times, discriminatory attitudes, and high cost of services. A combination of formal and informal healthcare services was common. The assessment of quality of care was closely related to undocumented immigration status. Social support networks are crucial to help solve healthcare issues. Quality of care should take intercultural health issues into account.
\end{abstract}

Reproductive Health; Quality of Health Care; Health Services; Emigrants and Immigrants

\section{Resumen}

El objetivo de este estudio fue conocer la experiencia de mujeres mexicanas migrantes en California, Estados Unidos, en torno a la utilización de los servicios formales de salud para resolver problemas relacionados con su salud sexual y reproductiva. El diseño fue cualitativo, con enfoque teórico metodológico de antropología interpretativa. Las técnicas utilizadas fueron historias de vida con mujeres usuarias de los servicios de salud en California y entrevistas breves con informantes clave. Se encontraron tres tipos de barreras principales para el acceso al sistema de salud: condición migratoria, idioma y género. Los tiempos de espera, actitudes discriminatorias y costo del servicio se expresaron como características que más incomodaron a las migrantes. La percepción de calidad de atención estuvo relacionada con la condición de ilegalidad migratoria. La red de apoyo tanto en México, como en California, colabora en la resolución de enfermedades. Se debe incorporar la perspectiva intercultural en los servicios.

Salud Reproductiva; Calidad de la Atención de Salud; Servicios de Salud; Emigrantes e Inmigrantes 


\section{Introducción}

Diversos estudios han documentado la situación de las mujeres mexicanas migrantes en Estados Unidos, en relación a los problemas a los que se enfrentan en el sistema sanitario de ese país en la búsqueda de atención a la salud 1,2,3,4. Cuando la condición migratoria es de ilegalidad, el problema se agrava, debido a que persiste el miedo a iniciativas de ley -como la 187-que prohibían el ingreso a los servicios públicos de personas ilegales y facultaba a los servidores públicos para denunciar a "sospechosos" de estar ilegalmente en Estados Unidos 5.

Se sabe que la condición legal (estar indocumentada o no) es decisiva para el acceso a los servicios públicos de salud, el miedo a ser deportado pesa más que el temor a padecer una enfermedad grave, esto se ha documentado, por ejemplo, en la atención de enfermedades como el VIH/SIDA 6,7.

En el caso de la atención a la salud sexual y reproductiva, tema de interés en la investigación que aquí se reporta, además de la situación migratoria la condición cultural influye en las barreras que limitan el acceso al sistema institucional de atención a la salud en Estados Unidos 8,9. Por ello, en este documento la salud sexual y reproductiva se entiende desde una perspectiva amplia, que incluye para su estudio las prácticas culturales y los espacios sociales en los que se recrea, además de los aspectos biológicos. En este sentido, en las zonas rurales de México, se ha explorado, por ejemplo, cómo las barreras culturales relacionadas con las creencias sobre la salud sexual y reproductiva influyen en la mortalidad materna, afectan la historia reproductiva de las mujeres por abortos mal practicados y condiciona el número de hijos que tienen 8 .

Sabiendo el arraigo de esas prácticas en México, se diseñó una investigación bilateral que exploró diversos aspectos relacionados con mujeres de origen mexicano que migran hacia Estados Unidos, particularmente al estado de California, ya que se calcula que junto con el estado de Texas concentran el 39\% de la migración latina indocumentada en Estados Unidos 10.

Uno de los aspectos explorados estuvo orientado en conocer ¿cómo resuelven los problemas de salud sexual y reproductiva mujeres mexicanas migrantes? ¿A qué barreras se enfrentan para acceder a los servicios formales de salud? y si ¿mantienen las prácticas culturales de su país de origen estando en un país diferente?

Estas preguntas cobran relevancia desde diferentes ópticas. Del lado teórico, es importante abundar sobre cómo en un contexto de alta vulnerabilidad social, mujeres migrantes utilizan diversas estrategias para atender su salud sexual y reproductiva, recreando elementos del lugar de origen que les permite no perder su identidad, lo cual ayuda a encontrarle sentido a su vida en otro país. Desde un punto de vista de la organización del sistema de salud, es relevante aportar información que contribuya a mejorar la calidad de la atención a la salud de mujeres migrantes, así como las posibilidades de abrir una nueva perspectiva en la atención que se les otorga.

\section{Método}

El objetivo de la investigación fue documentar mediante un estudio de caso en Yolo, condado de California, Estados Unidos, la experiencia de mujeres mexicanas migrantes indocumentadas, en torno a la utilización de los servicios de salud para resolver problemas relacionados con su salud sexual y reproductiva.

El diseño teórico-metodológico fue cualitativo-interpretativo 11,12,13. Se elaboraron historias de vida, siguiendo los supuestos de Daniel Bertaux, respecto al valor del relato biográfico para la comprensión de la subjetividad 14 .

La selección de los casos para elaborar las historias de vida representó un desafío metodológico, debido a la clandestinidad en la que viven las mujeres migrantes indocumentadas, lo cual es una situación que se sumó a la dificultad de abordar el tema de la vida sexual y reproductiva.

Considerando este contexto, el trabajo de campo se dividió en cuatro etapas.

En la primera, se capacitó a las personas que trabajarían recolectando información en el proyecto sobre aspectos éticos, necesarios para abordar temas como el de la migración clandestina. La segunda estuvo dedicada a realizar observación no participante en las clínicas y centros comunitarios en el condado de Yolo: Planned Parenthood en Sacramento y en Woodland; The Davis Community Clinic y el Yolo Family Resource Center en Woodland. Durante esta etapa también se identificaron informantes clave para ser entrevistados. En México, en el estado de Oaxaca (elegido por ser uno de los estados con más población migrante hacia Estados Unidos), participó una organización civil, identificando casos de profesionales formales y no formales de atención a la salud, que hubieran atendido a mujeres migrantes retornadas a sus localidades de origen por algún problema relacionado con su salud sexual y reproductiva. También se eligieron dos mujeres migrantes que estaban en tránsito en Oaxaca, esperando volver a California. En Yolo, se seleccionaron personas con una larga trayectoria en los servicios de apoyo a migrantes mexicanos, 
reconocidas por éstos como estratégicas en la resolución de sus problemas de salud. En la tercera etapa se diseñaron, imprimieron y repartieron folletos informativos durante seis meses en las clínicas antes mencionadas en Estados Unidos, para invitar al estudio a mujeres migrantes que quisieran narrar su experiencia. Todas las mujeres que respondieron fueron entrevistadas de manera breve para identificar a los casos más representativos. Quienes no fueron seleccionadas para elaborar las historias de vida y necesitaban apoyo médico, se las canalizó a diversas organizaciones que atienden migrantes para recibir servicios gratuitos. En la cuarta etapa se elaboraron ocho historias de vida, el criterio principal para elegir los casos fue el tiempo de residencia en Estados Unidos de manera ilegal. Las historias de vida se efectuaron mediante encuentros repetidos de entrevistas, realizados en espacios físicos solicitados a las autoridades de los centros que colaboraron en la investigación, para garantizar el anonimato de las participantes.

Se elaboraron guías temáticas para orientar la obtención de datos de las historias de vida con las mujeres que fueron seleccionadas. Para las entrevistas con los informantes clave se elaboraron guías de preguntas semi-estructuradas. La observación no participante requirió de guías de ejes de análisis.
Todos los diálogos con las informantes se grabaron en audio, previo consentimiento informado. Se transcribieron de manera literal y se crearon archivos en procesador de texto. La información obtenida se analizó manualmente, siguiendo la técnica de análisis del discurso ${ }^{15}$. Se elaboraron matrices de datos por categoría temática y por tipo de informante.

Los datos se recogieron durante 2010. A las participantes se les garantizó el anonimato y la confidencialidad en la información que reportaron, siguiendo los aspectos éticos de la investigación con sujetos humanos de la Declaración de Helsinki. El protocolo fue aprobado por la Comisión de Ética e Investigación de la Universidad de California en Davis, Estados Unidos.

\section{Resultados}

Se elaboraron ocho historias de vida (Tabla 1), la edad de las participantes osciló entre 20 y 45 años. Seis estaban casadas y dos eran solteras. Tres participantes tenían educación primaria y cinco secundaria. Dos habían vivido en Estados Unidos por 20 años o más, cuatro entre 10 y 19 años, y dos habían permanecido 10 años. Una de las participantes nació en Texas. Casi todas las participantes emigraron del centro de México.

Tabla 1

Características sociodemográficas de las participantes.

\begin{tabular}{|c|c|c|c|c|c|c|c|}
\hline $\begin{array}{l}\text { Número de } \\
\text { la entrevista }\end{array}$ & Edad & Número de hijos & Abortos & $\begin{array}{l}\text { Estado } \\
\text { civil }\end{array}$ & $\begin{array}{l}\text { Lugar de } \\
\text { nacimiento }\end{array}$ & $\begin{array}{l}\text { Año de migración a } \\
\text { los Estados Unidos }\end{array}$ & Escolaridad \\
\hline $\mathrm{C} 01$ & 29 & $\begin{array}{l}4 \text { (1 nacido en México y } \\
3 \text { en los Estados Unidos) }\end{array}$ & 1 & Casada & $\begin{array}{l}\text { Oaxaca, } \\
\text { México }\end{array}$ & 1997 & $\begin{array}{c}\text { Primaria } \\
\text { incompleta }\end{array}$ \\
\hline $\mathrm{CO} 2$ & 34 & $\begin{array}{c}1 \text { (nacida en los Estados } \\
\text { Unidos) }\end{array}$ & $\begin{array}{c}1 \text { espontáneo; } \\
1 \text { embarazo } \\
\text { ectópico }\end{array}$ & Casada & Jalisco, México & 2000 & Secundaria \\
\hline $\mathrm{CO3}$ & 45 & $\begin{array}{c}4 \text { (1 México y } 3 \text { en los } \\
\text { Estados Unidos) }\end{array}$ & 0 & Casada & Jalisco, México & 1988 & Secundaria \\
\hline $\mathrm{CO} 4$ & 20 & $\begin{array}{c}1 \text { (nacido en los Estados } \\
\text { Unidos) }\end{array}$ & 0 & Casada & $\begin{array}{l}\text { Michoacán, } \\
\text { México }\end{array}$ & 2000 & Primaria \\
\hline $\mathrm{CO5}$ & 34 & 0 & 0 & Soltera & Jalisco, México & 2004 & Secundaria \\
\hline $\mathrm{CO6}$ & 37 & $\begin{array}{l}2 \text { (nacidos en los } \\
\text { Estados Unidos con } \\
\text { discapacidad) }\end{array}$ & 0 & Casada & $\begin{array}{l}\text { Morelos, } \\
\text { México }\end{array}$ & 1998 & Secundaria \\
\hline $\mathrm{C08}$ & 32 & $\begin{array}{c}2 \text { (1 nacido en México y } \\
1 \text { nacido en los Estados } \\
\text { Unidos); Embarazada }\end{array}$ & 1 espontáneo & Casada & $\begin{array}{l}\text { Guanajuato, } \\
\text { México }\end{array}$ & 2002 & Primaria \\
\hline $\mathrm{CO9}$ & 42 & 4 hijos & 0 & Separada & $\begin{array}{l}\text { Michoacán, } \\
\text { México }\end{array}$ & 1989 & Secundaria \\
\hline
\end{tabular}


Siete tenían al menos un hijo. Una de las participantes estaba embarazada en el momento de la entrevista. El motivo de la migración hacia Estados Unidos fue buscar mejores condiciones de vida familiar. Menos uno de los casos, todas migraron para reunirse con alguno de sus familiares que ya radicaba en Estados Unidos. Las informantes salieron y entraron a Estados Unidos de manera ilegal en repetidas ocasiones, regresaron a sus localidades de origen por diferentes motivos, entre los que destaca la atención de enfermedades de ellas, de algún familiar cercano o por motivos religiosos. La mayoría de las casadas encontraron su pareja viviendo en Estados Unidos.

Respecto a las informantes clave (Tabla 2), se eligieron siete, representativos del problema que interesaba documentar. Dos seleccionadas en Yolo y cinco en Oaxaca. Las de Estados Unidos eran profesionales egresadas de Estudios Chicanos y trabajaban como promotoras de salud, estableciendo vínculos formales entre las instituciones públicas estadounidenses y las familias migrantes con más necesidades de apoyo gubernamental. Dos de Oaxaca eran parteras reconocidas por su larga trayectoria. Otro, médico especialista en ginecología encargado del área de salud reproductiva del hospital general de Oaxaca. Dos más eran mujeres migrantes que se encontraban en Oaxaca esperando regresar a Estados Unidos.

Para atender su salud sexual y reproductiva, las informantes enfrentaron barreras relacionadas con su condición migratoria, con el idioma y de género.
Sobre la condición migratoria, debido a que todas eran ilegales, a pesar de haber estado radicadas entre 10 y 20 años en el estado de California, no conocían el funcionamiento del sistema de salud estadunidense. Por ello, acudían a demandar servicios de atención a la salud en lugares del condado de Yolo, en los cuales se sabía a través de la red de apoyo que se atendía migrantes indocumentados de manera gratuita. Para la atención al parto elegían ir a la "clínica comunitaria", porque les proporcionaba mejor atención y había más personal de salud que hablaba español. Sin embargo, en la mayoría de las ocasiones en que se necesitaba un consejo relacionado con la salud, por ejemplo, el uso de métodos anticonceptivos, interrupciones del embarazo o enfermedades de transmisión sexual, la información provino de amigas, comadres y vecinas.

El idioma representó el obstáculo principal para comunicarse y utilizar los servicios de salud formales, ya que no solamente les impedía informarse sobre otros lugares a donde podían acudir a demandar el servicio, sino influía en su percepción respecto de la calidad de la atención médica recibida. Consideraban que el personal de salud que las atendía "medio entendían" español y no estaban seguras de que comprendieran sus dolencias, por ello, acudir a la clínica comunitaria no era su primera opción.

Al respecto, las informantes clave de Yolo señalaron que las clínicas que atienden personas indocumentadas, generalmente, contratan personal especializado y sensibilizado hacia la

Tabla 2

Informantes clave.

\begin{tabular}{|c|c|c|c|c|}
\hline Lugar de nacimiento & Sexo & Edad & Estudios & Ocupación \\
\hline California, Estados Unidos & Mujer & 26 & Licenciatura & $\begin{array}{l}\text { Radica en Yolo, California. Trabaja informando a familias migrantes en } \\
\text { California sobre los servicios que se proporcionan en la comunidad, } \\
\text { específicamente en el tema de salud. }\end{array}$ \\
\hline $\begin{array}{l}\text { Jalisco, México (residente en } \\
\text { Estados Unidos desde los } \\
6 \text { años) }\end{array}$ & Mujer & 27 & $\begin{array}{l}\text { Maestría en } \\
\text { Estudios Chicanos }\end{array}$ & $\begin{array}{l}\text { Radica en Yolo, California. Trabaja informando a familias migrantes sobre } \\
\text { servicios que se proporcionan en la comunidad en el tema de salud. Tramita }\end{array}$ \\
\hline Tlacolula, Oaxaca, México & Mujer & 68 & Primaria & Partera con larga trayectoria y reconocimiento local a su trabajo \\
\hline México D.F. & Mujer & 40 & Licenciatura & $\begin{array}{l}\text { Partera, líder de una organización de la sociedad civil que trabaja sobre } \\
\text { derechos reproductivos. }\end{array}$ \\
\hline Oaxaca, Oaxaca, México & Hombre & 34 & $\begin{array}{l}\text { Especialidad en } \\
\text { ginecología }\end{array}$ & Médico, ginecólogo especialista en el hospital general de Oaxaca. \\
\hline Tlacolula, Oaxaca, México & Mujer & 28 & Primaria incompleta & $\begin{array}{l}\text { En tránsito, en Oaxaca, esperando regresar a California y reunirse con su } \\
\text { esposo, quien es migrante ilegal en California. }\end{array}$ \\
\hline Ciudad de Oaxaca, México & Mujer & 31 & Primaria & $\begin{array}{l}\text { Con larga experiencia de vida como migrante ilegal en California, se } \\
\text { encontraba en Oaxaca, esperando regresar a reunirse con su familia. }\end{array}$ \\
\hline
\end{tabular}


atención a personas ilegales, sin embargo, la falta de comprensión del idioma y la ausencia de personal capacitado en asuntos de interculturalidad y salud, generan malos entendidos en la relación médico-paciente, los cuales se agrandan por el temor de las mujeres a ser identificadas como ilegales y eventualmente deportadas. Esto a pesar de no ser una práctica común en las clínicas es un rumor que se difunde entre los inmigrantes y les evita acudir abiertamente a pedir apoyo médico (Tabla 3).

El temor a la deportación se agranda, porque los tiempos para ser atendidas son largos, es frecuente esperar entre dos y cuatro horas para la consulta médica. En las clínicas de atención gratuita existen traductores, pero es difícil encontrarlos desocupados, debido a la alta demanda de atención médica de personas de origen latino. Otra de las informantes clave, que hace consejería con familias migrantes, señaló que esto es un hecho frecuente. Producto de la observación no participante fue corroborar que, a pesar de que exista personal de salud desocupado en la clínica, no se les atiende rápidamente. De hecho, cuando llegan no se les pregunta el motivo de la visita, las mujeres llegan, se sientan y esperan a que alguien les pregunte a qué van, son invisibles en la sala de espera. La falta de dominio del idioma dificulta transmitir incluso una emergencia obstétrica al personal de salud.

A los problemas sociales del acceso a los servicios de salud, se suman las barreras de género que se viven al interior del hogar. La cultura de género masculina se manifiesta fuera de México en eventos como estos, con frecuencia no les permitieron acudir a las consultas prenatales, puerperio, o para el control de su fecundidad. En esto se conjugaron varios aspectos: las creencias culturales de los varones respecto a cómo debe ser atendido un parto, el tema del uso de anticonceptivos y el temor a que la familia fuera identificada como ilegal. La violencia intrafamiliar también está presente, a pesar de que los servicios de las clínicas comunitarias en Yolo

Tabla 3

Cuadro comparativo de opiniones de los informantes clave sobre la atención a la salud sexual y reproductiva.

\begin{tabular}{|c|c|c|c|c|}
\hline Tema & $\begin{array}{c}\text { Proveedores informales: } \\
\text { parteras }\end{array}$ & $\begin{array}{l}\text { Proveedores formales: } \\
\text { médico }\end{array}$ & $\begin{array}{l}\text { Consejeras de familias de } \\
\text { migrantes en Estados Unidos }\end{array}$ & $\begin{array}{l}\text { Mujeres con experiencia de } \\
\text { migración en Estados Unidos }\end{array}$ \\
\hline $\begin{array}{l}\text { Acceso a servicios } \\
\text { formales e informales } \\
\text { de salud }\end{array}$ & $\begin{array}{l}\text { Las mujeres pueden } \\
\text { ir a los hospitales si } \\
\text { pertenecen al programa } \\
\text { de ayuda social } \\
\text { Oportunidades o Seguro } \\
\text { Popular, esto facilita la } \\
\text { atención del embarazo. } \\
\text { Atiende a las mujeres que } \\
\text { la buscan, ya sea en su } \\
\text { casa o donde las mujeres } \\
\text { decidan. Ellas van a las } \\
\text { comunidades cuando } \\
\text { solicitan su trabajo. } \\
\text { tienen acuerdos. } \\
\text { combarazo, deben referir } \\
\text { con el médico(a) que ya } \\
\text { comolicaciones del }\end{array}$ & $\begin{array}{l}\text { Las pacientes que } \\
\text { atienden llegan referidas } \\
\text { de los centros de salud } \\
\text { del programa de ayuda } \\
\text { social Oportunidades } \\
\text { o Seguro Popular. La } \\
\text { mayoría de casos que } \\
\text { atienden en Oaxaca son } \\
\text { de embarazos y abortos. }\end{array}$ & $\begin{array}{l}\text { Las mujeres migrantes prefieren } \\
\text { seguir las recomendaciones de las } \\
\text { personas cercanas para atender } \\
\text { problemas derivados del embarazo. } \\
\text { No les gusta ser atendidas por un } \\
\text { médico hombre. La mayoría no sabe } \\
\text { los diferentes tipos de servicios } \\
\text { médicos gratuitos que se ofertan y } \\
\text { tienen miedo del costo elevado de } \\
\text { la atención a la salud en Estados } \\
\text { Unidos. }\end{array}$ & $\begin{array}{l}\text { Tiene Seguro Popular y } \\
\text { reciben apoyo de programas } \\
\text { sociales como Oportunidades. } \\
\text { Acuden al centro de salud de } \\
\text { su localidad para atenderse, } \\
\text { para pedir información sobre } \\
\text { métodos de planificación } \\
\text { familiar y para los partos. }\end{array}$ \\
\hline
\end{tabular}

(continúa) 


\begin{tabular}{|c|c|c|c|c|}
\hline Tema & $\begin{array}{c}\text { Proveedores informales: } \\
\text { parteras }\end{array}$ & $\begin{array}{l}\text { Proveedores formales: } \\
\text { médico }\end{array}$ & $\begin{array}{c}\text { Consejeras de familias de } \\
\text { migrantes en Estados Unidos }\end{array}$ & $\begin{array}{l}\text { Mujeres con experiencia de } \\
\text { migración en Estados Unidos }\end{array}$ \\
\hline \multirow[t]{3}{*}{$\begin{array}{l}\text { Satisfacción en la } \\
\text { calidad de la atención }\end{array}$} & $\begin{array}{l}\text { Acuden a pedir consulta } \\
\text { mujeres de todo tipo } \\
\text { de condición social. } \\
\text { Consideran que a las } \\
\text { mujeres les gusta que les } \\
\text { pregunten si quieren parir } \\
\text { acostadas o sentadas. } \\
\text { Ellas las atienden como la } \\
\text { mujer prefiera. }\end{array}$ & $\begin{array}{c}\text { Consideran que los } \\
\text { tiempos de espera son } \\
\text { largos, se intenta hacer } \\
\text { consulta por cita, pero } \\
\text { esto todavía no funciona } \\
\text { bien. Creen que el tiempo } \\
\text { de espera es una de las } \\
\text { quejas principales de las } \\
\text { usuarias. }\end{array}$ & $\begin{array}{l}\text { Consideran que el problema } \\
\text { principal es el idioma, está } \\
\text { relacionado con los largos tiempos } \\
\text { de espera para ser atendidas, ya que } \\
\text { deben esperar por un traductor o } \\
\text { por el médico (a) que hable español, } \\
\text { lo cual genera incomodidad en las } \\
\text { mujeres, se sienten discriminadas. }\end{array}$ & $\begin{array}{l}\text { Les gusta como son atendidas } \\
\text { en los centros de salud. } \\
\text { A veces tienen problemas por } \\
\text { la lengua, los médicos (as) no } \\
\text { hablan la lengua local y en los } \\
\text { hospitales no hay traductores. } \\
\text { Sin embargo, deben acudir a la } \\
\text { misma clínica para mantener su } \\
\text { "tarjeta de gratuidad". }\end{array}$ \\
\hline & $\begin{array}{l}\text { Mujeres que ha referido } \\
\text { a clínicas de 2o nivel les } \\
\text { han dicho que la atención } \\
\text { es cara, debido a ello, } \\
\text { prefieren optar por la } \\
\text { atención de la partera. } \\
\text { La atención que } \\
\text { proporcionan parte del } \\
\text { respeto al cuerpo de la } \\
\text { mujer, tratan a sus usuarias } \\
\text { con calidad humana. }\end{array}$ & $\begin{array}{l}\text { Las mujeres que acuden } \\
\text { al servicio tienen } \\
\text { dificultades para ser } \\
\text { atendidas, debido a la } \\
\text { barrera del idioma, sin } \\
\text { embargo, lo resuelven } \\
\text { haciéndose acompañar } \\
\text { por algún familiar que } \\
\text { hable español, porque el } \\
\text { hospital no cuenta con } \\
\text { traductores. }\end{array}$ & & \\
\hline & $\begin{array}{l}\text { Acuden a su servicio hasta } \\
\text { que la mujer va a dar a } \\
\text { luz. Después de nacido } \\
\text { el bebé, dan información } \\
\text { sobre métodos de } \\
\text { Planificación Familiar. } \\
\text { En comparación con la } \\
\text { atención en el hospital, allí } \\
\text { deben ir con frecuencia y } \\
\text { les molesta que las "miren } \\
\text { los doctores". }\end{array}$ & $\begin{array}{l}\text { Las pacientes indígenas } \\
\text { siguen mejor las } \\
\text { indicaciones que las de } \\
\text { zona urbana. Acuden } \\
\text { regularmente a la consulta } \\
\text { prenatal. Siete de cada } \\
10 \text { mujeres no aceptan } \\
\text { utilizar un método } \\
\text { anticonceptivo. }\end{array}$ & & \\
\hline
\end{tabular}

(continúa) 


\begin{tabular}{|c|c|c|c|c|}
\hline Tema & $\begin{array}{c}\text { Proveedores informales: } \\
\text { parteras }\end{array}$ & $\begin{array}{l}\text { Proveedores formales: } \\
\text { médico }\end{array}$ & $\begin{array}{c}\text { Consejeras de familias de } \\
\text { migrantes en Estados Unidos }\end{array}$ & $\begin{array}{l}\text { Mujeres con experiencia de } \\
\text { migración en Estados Unidos }\end{array}$ \\
\hline $\begin{array}{l}\text { Reproducción de } \\
\text { prácticas culturales } \\
\text { de su país de origen, } \\
\text { respecto a la atención } \\
\text { de la salud }\end{array}$ & $\begin{array}{l}\text { Les dan remedios } \\
\text { preparados con plantas } \\
\text { medicinales y les dicen } \\
\text { como tomarlos. Es } \\
\text { frecuente que las mujeres } \\
\text { acudan con las parteras } \\
\text { para pedírselos. }\end{array}$ & $\begin{array}{l}\text { Las mujeres no hacen } \\
\text { seguimiento a su } \\
\text { procedimiento de aborto } \\
\text { o parto, porque creen que } \\
\text { ya no es necesario una vez } \\
\text { que salen de la clínica. No } \\
\text { usan Planificación Familiar. }\end{array}$ & $\begin{array}{c}\text { Si no hay complicaciones en el } \\
\text { parto, la mayoría no regresa a la } \\
\text { clínica para seguirse atendiendo, el } \\
\text { puerperio se pasa en casa, siguiendo } \\
\text { las recomendaciones de la familia y } \\
\text { amistades cercanas. } \\
\text { En California existen parteras, } \\
\text { curanderas, sobadoras que ejercen } \\
\text { su práctica. Las mujeres se enteran } \\
\text { por amistades, familia, vecinos. } \\
\text { Es común que se les visite para } \\
\text { atenderse de problemas derivados } \\
\text { durante el embarazo y puerperio, } \\
\text { también para indagar información } \\
\text { sobre métodos anticonceptivos. } \\
\text { Estas prácticas se realizan } \\
\text { independientemente de que acudan } \\
\text { a la clínica. }\end{array}$ & $\begin{array}{l}\text { Utilizan plantas medicinales } \\
\text { y sus familiares prefieren } \\
\text { atenderse con la partera. } \\
\text { Cuando se trata de la } \\
\text { atención al parto siguen las } \\
\text { recomendaciones de la familia, } \\
\text { pero también acuden al médico } \\
\text { para asegurarse que el bebé } \\
\text { está bien. }\end{array}$ \\
\hline
\end{tabular}

también contemplan consejería e intervención en casos de crisis por violencia con la pareja, la barrera del idioma impide el acercamiento a esas instancias. Debido a ello, en ocasiones este problema se agrega a su vulnerabilidad social como migrantes ilegales (Tabla 4).

Estas barreras se relacionan con la percepción sobre la satisfacción en la calidad de la atención con que son atendidas. Las informantes clave, parteras de México, señalaron haber atendido mujeres migrantes embarazadas, que habían regresado a sus localidades solamente para atenderse el parto, debido a que las mujeres prefieren pasar ese evento con personas con las que encontrarán una consonancia cultural.

La experiencia de estas mujeres por los servicios de salud que utilizaron en Yolo ocurrió bajo la percepción continua de ser discriminadas, debido a su condición migratoria, lo cual las desalentó para volver a buscar atención médica. En parte por ello algunas señalaron haber regresado a México para ser atendidas, a pesar de correr el riesgo de no volver a entrar a Estados Unidos. Las informantes clave de Yolo, consideraron que la discriminación de la que son objeto es una de las razones por las cuales las mujeres migrantes prefieren buscar el apoyo de redes informales de atención a la salud en el condado, en donde también existen parteras, sobadoras y curanderas emigradas, con conocimientos de plantas medi- cinales que ayudan a paliar síntomas. También es común que utilicen medicamentos que se piden a familiares en México (Tabla 5).

La percepción de la calidad de la atención mejoró cuando las mujeres se atendieron en clínicas en Yolo que cuentan con parteras profesionales (midwifes). En estos casos se percibe mayor calidez en la consulta, existe más confianza, debido a que relacionan ese trabajo con el de las parteras empíricas en México.

Otra percepción negativa que influye en la demanda de atención médica es el rumor sobre el costo de la atención; este aspecto se señaló de manera reiterada. Por ello, además de la asesoría médica con la red de apoyo cercana, se realizan consultas médicas por teléfono con los doctores en las localidades de origen en México y se recibe el medicamento vía postal. A pesar de que ninguna de las informantes se había enfrentado a un gasto excesivo para atender sus partos, pérdidas de embarazo o consulta para planificación familiar, enfermarse de gravedad era un temor frecuente.

Otro aspecto que les generaba estrés después de acudir al médico era el costo de los medicamentos prescritos. Por ello, con frecuencia los adquirían de manera clandestina, entre los migrantes indocumentados se sabía en qué farmacia del condado se podía acudir para comprarlos sin receta. 
Barreras para el acceso a los servicios formales de atención a la salud.

\begin{tabular}{|c|c|}
\hline Tipo de barrera & Situaciones a las que se enfrentan \\
\hline \multirow[t]{5}{*}{ Ilegalidad migratoria } & Deben acudir a los servicios de salud que prestan las instituciones no lucrativas. \\
\hline & $\begin{array}{l}\text { Prefieren el apoyo de parteras y curanderas de origen mexicano, residentes en Estados Unidos, para recibir atención } \\
\text { en el parto y pedir información sobre salud sexual y reproductiva. }\end{array}$ \\
\hline & No pueden tener un número de seguro social, lo cual les impide moverse en los terrenos formales del país. \\
\hline & Les falta información sobre las posibilidades que tienen para atender su salud sexual y reproductiva. \\
\hline & $\begin{array}{l}\text { "Trafican" con medicamentos, les resulta más barato y confiable realizar una transacción con empleados (as) de } \\
\text { farmacias en Estados Unidos que les proporcionan medicinas para controlar algún malestar, que enfrentar la cita } \\
\text { médica y exponerse a cuestionamientos sobre su condición migratoria. }\end{array}$ \\
\hline \multirow[t]{2}{*}{ Idioma } & No pueden comunicarse con el personal de salud, lo cual les genera inseguridad sobre estar bien atendidas. \\
\hline & $\begin{array}{l}\text { En ocasiones deben esperar que llegue el traductor a la clínica para que puedan ser atendidas, o a que el médico (a) } \\
\qquad \text { que habla español se desocupe. }\end{array}$ \\
\hline \multirow[t]{2}{*}{ De género } & $\begin{array}{l}\text { Se enfrentan a situaciones de control de su salud reproductiva, ya que es frecuente que los esposos decidan si van al } \\
\text { médico(a) y si utilizan métodos anticonceptivos. }\end{array}$ \\
\hline & Violencia intrafamiliar. \\
\hline
\end{tabular}

Utilización de recursos informales de salud para atender problemas de salud sexual y reproductiva.

\begin{tabular}{|c|c|c|c|}
\hline & Parteras & Curanderas & Sobadoras \\
\hline \multirow{7}{*}{$\begin{array}{l}\text { ¿En qué casos se } \\
\text { utiliza? }\end{array}$} & Atención de partos. & Empacho. & Acomodar el producto. \\
\hline & Búsqueda de medicina & Mal de ojo. & Conocer el sexo del \\
\hline & alternativa. & & producto. \\
\hline & & Preparados de plantas medicinales & \\
\hline & Información sobre & para malestares durante el embarazo. & Curar sangrado menstrual \\
\hline & métodos anticonceptivos. & Interrupción de embarazos. & excesivo. \\
\hline & & & Problemas de infertilidad. \\
\hline
\end{tabular}

\section{Discusión}

En la reproducción de las prácticas culturales de atención a la salud en los contextos de migración ilegal, convergen diversos aspectos de la cultura de origen que responden a las preguntas orientadoras de la investigación que aquí se reporta. Los problemas de salud sexual y reproductiva son resueltos mediante la utilización, tanto de los recursos formales de atención a la salud, como de los informales, aunque estos últimos son a los que más se acude por el temor a ser deportadas. Este hallazgo coincide con lo reportado por estudios como el de Marshall et al. 10.

De las barreras a las que se enfrentan destaca el idioma como el principal obstáculo para acudir a demandar servicios de salud, además de que la falta de entendimiento, promueve un comportamiento defensivo. Por ello, llama la atención la búsqueda de profesionales no for- 
males para atenderse, a pesar de estar en otro país ${ }^{16}$, en esa búsqueda destaca la movilidad que las informantes tuvieron en el interior de Estados Unidos para acceder a ese tipo de ayuda a pesar de su condición migratoria.

La estructura del sistema de salud estadounidense permite la atención de personas ilegales, a través de la atención en clínicas que otorgan atención médica gratuita, como parte de una labor altruista. De acuerdo a la experiencia de las informantes, esa gratuidad del servicio se proporciona bajo condiciones de mala calidad en la atención médica, similares a las que viven mujeres con la misma condición socioeconómica en México 2,7.

Las barreras de acceso para la utilización de los servicios tienen relación con una subjetividad construida alrededor de la vulnerabilidad que les da la condición de migrantes ilegales, ésta promueve el temor a ser deportadas, evita demandar información sobre el padecimiento, sobre el costo del servicio, de los medicamentos y de otras opciones de atención médica 17.

Es relevante advertir que en el contexto actual de alta migración trasnacional el tema de la atención a la salud representa un problema global grave, debido a que la demanda de atención de los servicios debe responder a la diversidad cultural a la que se enfrentan los países, lo cual implica adecuaciones del sistema de salud. Ortega et al. 18 encontraron que la utilización de recursos de atención médica de migrantes latinos es muy baja, al compararse con latinos nacidos en Estados Unidos. Estos hallazgos son similares a los de Marshall et al. 10 con inmigrantes en Texas, quienes además de lo aquí mencionado abundan sobre la importancia de que en Estados Unidos deben promoverse mayores oportunidades de acceso al sistema de salud de las personas migrantes. Otros autores 19, además, encontraron que el miedo de los inmigrantes ilegales a ser deportados atrasa la búsqueda de atención a la salud, agravando la enfermedad y promovien- do prácticas clandestinas para comprar medicamentos y atenderse, dos de los aspectos también hallados en la investigación aquí reportada.

Por otro lado, la percepción de la "necesidad" está determinada por el horizonte cultural de las mujeres. Esto explica la razón de la elección de los servicios informales de salud, a pesar de tener las clínicas de atención médica gratuita en Estados Unidos, es un hecho que diversos estudios han señalado y que también está relacionado con la capacidad de agencia, es decir, de las posibilidades que las personas tienen para allegarse de recursos materiales o humanos cuando necesitan resolver un problema 20,21.

La participación de la red de apoyo social 22 en la búsqueda de recursos alternativos de salud es un signo de resistencia cultural y una muestra de cómo se sobrevive en los ambientes de ilegalidad migratoria. Sobre este punto opera un aspecto negativo, la movilidad de las informantes y la búsqueda de los recursos informales no promueve un acercamiento sostenido con el personal de salud de las clínicas, con lo cual no se puede construir una relación médico-paciente satisfactoria. En el caso de las mujeres embarazadas, esto evita que tengan un control prenatal adecuado, poniendo en riesgo la salud tanto de la madre como del producto. La falta de control prenatal es uno de los problemas que se ha relacionado con la mortalidad materna 23 .

El asunto de la diversidad cultural 5,6,18 representa un reto para los servicios de salud y para la biomedicina. Los hallazgos aquí presentados tienen relevancia para el personal de salud, los tomadores de decisiones encargados de formular las políticas de salud pública y para los grupos de abogacía y defensa de migrantes que trabajan en ambos lados de la frontera, quienes consideran que las mujeres inmigrantes - independientemente de su condición legal - tienen derecho a acceder a servicios de salud reproductiva de calidad. 


\section{Resumo}

O objetivo deste estudo foi conhecer a experiência de mulheres imigrantes mexicanas na Califórnia, Estados Unidos, sobre a utilização de serviços formais de saúde para resolver problemas relacionados com a saúde sexual e reprodutiva. O desenho foi qualitativo, com enfoque teórico-metodológico da Antropologia Interpretativa. As técnicas utilizadas foram relatos de histórias de vida de mulheres usuárias dos serviços de saúde na Califórnia e entrevistas breves com informantes-chave. Encontraram-se três tipos de barreiras principais para o acesso ao serviço de saúde: condições de imigração idioma e gênero. Tempo de espera, atitudes discriminatórias e custo do serviço foram as características que mais incomodaram as imigrantes. A percepção de qualidade da atenção esteve relacionada com a condição de ilegalidade migratória. A rede de apoio, tanto no México quanto na Califórnia, colabora na resolução das enfermidades. Deve-se incorporar a perspectiva intercultural nos serviços de saúde.

Saúde Reprodutiva; Qualidade da Assistência à Saúde Serviços de Saúde; Emigrantes e Imigrantes

\section{Colaboradores}

N. Deeb-Sossa contribuyó con el diseño de la investigación en los Estados Unidos y aprobación final de la versión que debe ser publicada. C. D. Olavarrieta contribuyó con el diseño de la investigación en México y aprobación final de la versión que debe ser publicada. C. Juárez-Ramírez contribuyó con la redacción del documento, revisión crítica del contenido intelectual y asesoría metodológica. S. G. García contribuyó con la revisión crítica del manuscrito, diseño del estudio y aprobación final de la versión que debe ser publicada. A. Villalobos contribuyó con el análisis de los datos y aprobación final de la versión que debe ser publicada.

\section{Referencias}

1. Caballero M, Leyva-Flores R, Ochoa-Marín SC Zarco A, Guerrero C. Las mujeres que se quedan: migración e implicación en los procesos de búsqueda de atención de servicios de salud. Salud Pública Méx 2008; 50:241-50.

2. Castañeda NS. Dinámica y proceso de migración a Estados Unidos: jóvenes de Guadalajara, Jalisco, México. Rev Latinoam Cienc Soc Niñez Juv 2009; 7:1459-90.

3. Arzate J, Vizcarra I. De la migración masculina trasnacional: violencia estructural y género en comunidades campesinas del estado de México. Migración y Desarrollo 2007; (9):95-112.

4. Salgado N, González T, Bojorquez I, Infante C. Vulnerabilidad social, salud y migración MéxicoEstados Unidos. Salud Pública Méx 2007; 49:8-10.

5. Marc LB, Schur CL. The effect of fear on access to care among undocumented Latino immigrants. J Immigr Health 2001; 3:151-6.
6. Hernández-Rosete D. La otra migración, historias de discriminación de personas que vivieron con VIH en México. Salud Mental 2008; 31:253-60.

7. Salgado N, González T, Infante C, Márquez M, Pelcastre B, Serván E. Servicios de salud en la Mixteca: utilización y condición de afiliación en hogares de migrantes y no-migrantes a Estados Unidos. Salud Pública Méx 2010; 52:424-31.

8. Sánchez-Domínguez MS, Leyva R, Caballero M, Infante C. Disposición a usar condón en localidades con alta movilidad poblacional de México y Centro América. Migración y Desarrollo 2010; 7:155-78.

9. Guadarrama X, Vizcarra I, Lutz B. De la migración ausencias masculinas y reacciones femeninas mazahuas. Relaciones 2009; XXX:183-219.

10. Marshall KJ, Urrutia-Rojas X, Soto F, Coggin C. Health status and access to health care of documented and undocumented immigrant Latino women. Health Care Women Int 2005; 26:916-36 
11. Denzin N. Un punto de vista interpretativo. In: Denman C, Haro J, editores. Por los rincones. Hermosillo: El Colegio de Sonora; 2000. p. 147-205.

12. Patton M. Qualitative research and evaluation methods. Thousand Oaks: Sage; 2002.

13. Coffey A, Atkinson P. Making sense of qualitative data: complementary research strategies. Thousand Oaks: Sage; 1996.

14. Bertaux D. Los relatos de vida. Barcelona: Ediciones Bellaterra; 2005.

15. Wetherell M, Potter J. El análisis del discurso y la identificación de los repertorios interpretativos. In: Gordo A, Linaza J, editores. Psicologías, discursos y poder. Madrid: Editorial Visor; 1996. p. 63-78.

16. Murray C, Kawabata K, Valentine N. People's experiences versus people's expectations. Health Aff $2001 ; 20: 21-4$

17. Nazroo J. The structuring of ethnic inequalities in health: economic position, racial discrimination and racism. Am J Public Health 2003; 93:277-84.

18. Ortega A, Fang H, Perez VH, Rizzo JA, CarterPokras O, Wallace SP, et al. Health care access, use of services, and experiences among undocumented Mexicans and other Latinos. Arch Intern Med 2007; 167:2354-60.
19. Berk ML, Schur CL, Chavez LR, Frankel M. Health care use among undocumented immigrants. Health Aff 2000; 19:51-65.

20. Kaplan CP, Erickson PI, Stewart SL, Crane LA. Young Latinas and abortion: the role of cultural factors, reproductive behavior, and alternative roles to motherhood. Health Care Women Int 2001; 22:667-89.

21. Lee JH, Goldstein MS, Brown RC, Ballard-Barbash R. How does acculturation affect the use of complementary and alternative medicine providers among Mexican- and Asian-Americans? J Immigr Minor Health 2010; 12:302-9.

22. Dressler WW, Balieiro MC, Dos Santos JE. The cultural construction of social support in Brazil: associations with health outcomes. Cult Med Psychiatry 1997; 21:303-35.

23. Freyermuth G. Las mujeres de humo. Morir en Chenalhó: género, etnia y generación, factores constitutivos del riesgo durante la maternidad. México DF: Centro de Investigaciones y Estudios Superiores en Antropología Social/Editorial Porrúa; 2003

Recibido el 08/Oct/2012

Aprobado el 08/Ene/2013 